\title{
Optical analogue of population trapping in the continuum: classical and quantum interference effects
}

\author{
Stefano Longhi \\ Dipartimento di Fisica and Istituto di Fotonica e Nanotecnologie del CNR, \\ Politecnico di Milano, Piazza L. da Vinci 32, I-20133 Milano, Italy
}

\begin{abstract}
A quantum theory of light propagation in two optical channel waveguides tunnelling-coupled to a common continuum of modes (such as those of a slab waveguide) is presented, and classical and quantum interference effects are investigated. For classical light, the photonic system realizes an optical analogue of coherent population trapping in the continuum encountered in atomic physics, where destructive interference between different light leakage channels leads to the appearance of a trapped state embedded in the continuum. For nonclassical light, two-photon interference effects are predicted, such as the tendency of photon pairs to bunch when decaying into the continuum.
\end{abstract}

PACS numbers: 42.82.Et, 42.50.Dv, 72.20.Ht

\section{INTRODUCTION}

It is well established that quantum mechanics of a nonrelativistic particle and paraxial wave optics in dielectric media shear strong formal similarities (see, for instance, [1-3]). Owing to such similarities, the temporal evolution of a quantum particle (e.g. an electron in an atom or in a crystal) can be mimicked by means of light propagation in suitably-designed photonic structures. Quantumoptical analogies have seen in recent years a renewed and increasing interest, both theoretically and experimentally, mainly motivated by the possibility offered by optics to visualize at a macroscopic level certain coherent phenomena, originally proposed for quantum systems, which may be of difficult access or of controversial interpretation in the quantum context (see, e.g., [4, 5] and references therein). In particular, recent works theoretically proposed and experimentally demonstrated the optical analogues of some important coherent phenomena encountered in atomic and molecular physics, such as coherent population transfer [6, 7], electromagneticallyinduced transparency [8], and population trapping in the continuum 9 11. The latter effect has been extensively investigated in atomic physics in connection, for instance, with the problem of autoionization of an atom by ultraviolet radiation or in laser-induced continuum structures (see [12, 13] and references therein). In the simplest case, population trapping in the continuum describes decay suppression of two discrete states coupled to a common and unstructured continuum: as a single bound state coupled to the continuum decays in an irreversible way and population is transferred into the continuum, under certain conditions coupling of the continuum with the additional other bound state may partially or totally suppress the decay of states owing to a destructive interference effect which was first studied by Fano for radiative transitions to autoionizing states in atoms 14. Fano-like resonances in photonic systems have been considered in several recent works as well [15, 16], with a main focus on the scattering (transmission) properties of the structures. These previous studies in optics have mostly con- sidered propagation of classical light in coupled guiding structures, disregarding the quantum nature of light. For classical light, destructive interference among different tunneling paths is responsible for the existence of bound states in the continuum, similarly to Fano interference in atomic physics. However, when few photons or nonclassical beams are used to excite the photonic structures, light propagation may show interference effects of quantum nature. Since long time, coupled waveguides are known to behave similarly to beam splitters (see, for instance, [17]) and to show strictly quantum features when nonclassical light propagates through them, such as twophoton Hong-Ou-Mandel quantum interference originally demonstrated for beam splitters [18]. Like beam splitters 19], coupled waveguides offer the possibility to transfer nonclassical properties of light and to generate entangled states [17, 20]. With recent technological advances in the realization of high-quality low-loss integrated photonic structures and nonclassical light sources, such possibilities are nowadays realities. High-fidelity Hong-OuMandel quantum interference and integrated optical realizations of other key quantum photonic circuits have been recently demonstrated in silicon-based waveguide couplers [21]. In another recent experiment, Bromberg et al. 22 showed nontrivial photon correlations in coupled waveguide arrays and observed them using classical intensity correlation measurements. The possibility offered by experimentalists to test quantum aspects of light in integrated optic networks motivates to extend previous studies of quantum-optical analogies from the classical to the quantum level, highlighting interference effects of purely quantum nature. For instance, a recent theoretical study of optical Bloch oscillations and Zener tunneling in optical lattices [23] showed that propagation of nonclassical light in the lattice may reveal certain particle-like aspects of light and quantum interference phenomena. In this work we investigate classical and quantum interference effects of light in an optical analogue of coherent population trapping, based on two optical channel waveguides side coupled to a common slab waveguide recently proposed in Ref.[9]. For classical light (coherent 
states), such system realizes in optics a simple analogue of destructive Fano interference with suppression of light leakage from the channel waveguides (analogous to two discrete states) into the slab waveguide (the analogue of the continuum). When the description of light propagation is made at the quantum level, photon pair excitation reveals strictly quantum features, such as the tendency of photons to bunch when decaying into the continuum.

\section{THE OPTICAL ANALOGUE OF POPULATION TRAPPING IN THE CONTINUUM: BASIC MODEL AND CLASSIC WAVE OPTICS DESCRIPTION}

\section{A. The model}

Let us consider propagation of quasi-monochromatic and paraxial light wave packets at carrier frequency $\omega=2 \pi c_{0} / \lambda$ in a weakling guiding dielectric structure, with optical axis $z$ and refractive index profile $n(x, y)$, composed by two straight and parallel single-mode and equal channel waveguides $\mathrm{W}_{1}$ and $\mathrm{W}_{2}$, side-coupled to a slab waveguide $\mathrm{S}$ as shown in Fig.1(a). In the paraxial, weak guidance and quasi-monochromatic approximations, the electric field can be written as $E(x, y, z, t) \propto$ $\psi(x, y, z, t) \exp (i \beta z-i \omega t)+\psi^{\dagger}(x, y, z, t) \exp (-i \beta z+i \omega t)$, where the envelope $\psi$ varies slowly with respect to $z$ and $t$ over one wavelength $\lambda$ and one optical cycle $2 \pi / \omega$. Neglecting nonlinearities and group-velocity dispersion, the slow evolution of the electric field envelope $\psi(x, y, z, t)$ along the paraxial $z$ direction is governed by the scalar wave equation

$$
i\left(\psi_{z}+\frac{1}{v_{g}} \psi_{t}\right)=-\frac{1}{2 \beta}\left(\psi_{x x}+\psi_{y y}\right)+V(x, y) \psi,
$$

where $\beta=\left(\omega / c_{0}\right) n_{s}$ is the reference propagation constant, $n_{s}$ is the substrate refractive index, $v_{g}=$ $(d \beta / d \omega)^{-1}$ is the group velocity of light, and $V(x, y)=$ $\beta\left[n_{s}-n(x, y)\right] / n_{s}$. The electric field envelope $\psi$ has been normalized such that the cycle-averaged total energy of the electromagnetic field (see, e.g., 24]) is given by

$$
U \simeq \frac{\hbar \omega}{v_{g}} \int d x d y d z \psi^{\dagger} \psi=\hbar \omega \int d x d y d t \psi^{\dagger} \psi
$$

At the entrance plane $z=0$, light is typically injected into either one, or in both, the channel waveguides $\mathrm{W}_{1}$ and $\mathrm{W}_{2}$, in the form of either monochromatic waves or wave packets with spatial profiles tailored to match their fundamental modes. Owing to evanescent coupling with the slab waveguide $\mathrm{S}$, light leakage into the continuous set of modes of the slab is generally found, however for certain geometric settings a trapping state may exist, corresponding to destructive interference between different tunneling paths into the continuum [9]. At a classical level, light propagation in the waveguides is well captured in the framework of a coupled mode equation approach, as previously shown in Ref.[9]. After introduction of the spectral decomposition $\psi(x, y, z, t)=$ $(2 \pi)^{-1 / 2} \int d \Omega \phi(x, y, z, \Omega) \exp (-i \Omega t)$, from Eq.(1) it follows that the spectral amplitude $\phi(x, y, z, \Omega)$ satisfies the wave equation

$$
i \phi_{z}=-\frac{1}{2 \beta}\left(\phi_{x x}+\phi_{y y}\right)+V(x, y) \phi-\frac{\Omega}{v_{g}} \phi .
$$

Let us indicate by $u_{1}(\rho)$ and $u_{2}(\rho)$ the fundamental modes of waveguides $\mathrm{W}_{1}$ and $\mathrm{W}_{2}$, and by $u_{k}(\rho)$ the continuous set of modes of the slab waveguide $\mathrm{S}$ with the normalization conditions $\int d \rho\left|u_{1}(\rho)\right|^{2}=\int d \rho\left|u_{2}(\rho)\right|^{2}=$ 1, $\int d \rho u_{k}(\rho) u_{k^{\prime}}^{*}(\rho)=\delta\left(k-k^{\prime}\right)$ and $\int d \rho u_{1}(\rho) u_{2}^{*}(\rho) \simeq$ $\int d \rho u_{1}(\rho) u_{k}^{*}(\rho) \simeq \int d \rho u_{2}(\rho) u_{k}^{*}(\rho) \simeq 0$, where $\rho \equiv(x, y)$. Let us search for a solution to Eq.(3) in the form

$$
\begin{aligned}
\phi(\rho, z, \Omega) & =\left[c_{1}(z, \Omega) u_{1}(\rho)+c_{2}(z, \Omega) u_{2}(\rho)+\right. \\
& \left.+\int d k c_{k}(z, \Omega) u_{k}(\rho)\right] \exp \left(i \Omega z / v_{g}\right) .
\end{aligned}
$$

The evolution equations of modal amplitudes $c_{1}, c_{2}$ and $c_{k}$ then read [9]

$$
\begin{aligned}
& i \frac{\partial c_{1}}{\partial z}=\Delta \beta_{0} c_{1}+\int d k g_{1}(k) c_{k} \\
& i \frac{\partial c_{2}}{\partial z}=\Delta \beta_{0} c_{2}+\int d k g_{2}(k) c_{k} \\
& i \frac{\partial c_{k}}{\partial z}=\Delta \beta(k) c_{k}+g_{1}^{*}(k) c_{1}+g_{2}^{*}(k) c_{2}
\end{aligned}
$$

where: $g_{1}(k)$ is the coupling amplitude between modes $u_{1}$ and $u_{k} ; g_{2}(k)$ is the coupling amplitude between modes $u_{2}$ and $u_{k}$; and $\Delta \beta(k), \Delta \beta_{0}$ are the propagation constant shifts of modes $u_{k}$ and $u_{1,2}$, respectively, from the reference value $\beta$. In their present form, Eqs.(5-7) are analogous to the dynamical equations describing the quantum mechanical decay of two bound states $\mathrm{W}_{1}$ and $\mathrm{W}_{2}$ into a common continuum S [see Fig.1(b)], provided that the temporal dynamics of the quantum mechanical problem is replaced by the paraxial propagation in space of light waves. The two bound states, with the same energy, are embedded in the continuum provided that $\Delta \beta_{0}$ falls inside the continuous spectrum $\Delta \beta(k)$, a condition which is satisfied whenever the refractive index change $\Delta n_{S}$ in the slab waveguide is smaller than the index change $\Delta n_{q}$ in the two channel waveguides (see [9] for more details). Note that, if waveguides $\mathrm{W}_{1}$ and $\mathrm{W}_{2}$ are symmetrically placed at opposite sides from the slab waveguide $\mathrm{S}$ as shown in Fig.1(a), the coupling coefficients $g_{1}(k)$ and $g_{2}(k)$ are the same, i.e. $g_{2}(k)=g_{1}(k) \equiv g(k)$, and in this case a trapped state does exist, as discussed in Ref. [9] Although trapped states in the continuum may also exist when the waveguides $\mathrm{W}_{1}$ and $\mathrm{W}_{2}$ are horizontally displaced or placed at different distances from the slab waveguide [9], in this work we will consider the simplest symmetric case shown in Fig.1(a). 


\section{B. Optical analogue of population trapping}

If one of the two channel waveguides, say e.g. $\mathrm{W}_{2}$, were removed, light initially injected into waveguide $\mathrm{W}_{1}$ would decay into the slab waveguide, a phenomenon fully analogous to the quantum mechanical decay of a bound state coupled to a continuum. In the markovian approximation, valid for weak coupling and for a nearly unstructured continuum, the decay is well described by an exponential law. The presence of waveguide $\mathrm{W}_{2}$ generally modifies the decay behavior and, under certain conditions, the decay can be suppressed owing to a destructive Fano-like interference of different decay channels. Full or fractional suppression of the decay is related to the appearance of a trapped (or dark) state in the continuum. To derive the decay laws of light waves in the channel waveguides $\mathrm{W}_{1}$ and $\mathrm{W}_{2}$, we follow a standard procedure [13], detailed for instance in Ref.[9], and eliminate the amplitudes $c_{k}$ of continuous modes by a formal integration of Eq.(7) with the initial condition $c_{k}(0, \Omega)=0$. This yields a set of two coupled integro-differential equations for the amplitudes $c_{1}$ and $c_{2}$ of discrete modes. In the weak coupling limit and assuming a nearly unstructured continuum, the markovian approximation can be made and the following reduced equations for amplitudes $c_{1}$ and $c_{2}$ are derived

$$
\frac{\partial c_{1}}{\partial z}=-i \Delta \beta_{0} c_{1}-\sigma\left(c_{1}+c_{2}\right), \quad \frac{\partial c_{2}}{\partial z}=-i \Delta \beta_{0} c_{2}-\sigma\left(c_{1}+c_{2}\right),
$$

where

$$
\sigma=\int_{0}^{\infty} d \tau \int d k|g(k)|^{2} \exp \left\{-i\left[\Delta \beta(k)-\Delta \beta_{0}\right] \tau\right\}
$$

is the decay rate of the single channel waveguide into the continuum. The solution to Eqs.(8) reads explicitly

$$
\begin{aligned}
& c_{1}(z, \Omega)=S_{11}(z) c_{1}(0, \Omega)+S_{12}(z) c_{2}(0, \Omega) \\
& c_{2}(z, \Omega)=S_{21}(z) c_{1}(0, \Omega)+S_{22}(z) c_{2}(0, \Omega)
\end{aligned}
$$

where

$$
\begin{aligned}
& S_{11}(z)=\frac{1}{2} \exp \left(-i \Delta \beta_{0} z\right)[1+\exp (-2 \sigma z)] \\
& S_{12}(z)=-\frac{1}{2} \exp \left(-i \Delta \beta_{0} z\right)[1-\exp (-2 \sigma z)] \\
& S_{22}(z)=S_{11}(z), \quad S_{21}(z)=S_{12}(z)
\end{aligned}
$$

Note that, in the quasi-monochromatic approximation assumed in this work, the matrix coefficients $S_{n, l}$ are independent of frequency $\Omega$. To understand the appearance of the optical analogue of population trapping, let us consider the monochromatic case, with the only nonvanishing spectral component at $\Omega=0$, and two different input excitations, corresponding the former to single waveguide excitation and the latter to simultaneous excitation of the two channel waveguides. In the former case, assuming for instance $c_{1}(0, \Omega)=\sqrt{2 \pi} \delta(\Omega)$ and $c_{2}(0, \Omega)=0$, one obtains

$\psi(\rho, z)=S_{11}(z) u_{1}(\rho)+S_{12}(z) u_{2}(\rho)+S_{13}(z) \theta(\rho, z) \quad(15)$ where the last term on the right hand side of Eq.(15) accounts for the light field tunnelled into the slab waveguide and $\theta(\rho, z)$ ia a suitable superposition of continuous modes $u_{k}(\rho)$ normalized such that $\int d \rho|\theta(\rho, z)|^{2}=1$. For power conservation, the relation $\left|S_{11}\right|^{2}+\left|S_{12}\right|^{2}+\left|S_{13}\right|^{2}=$ 1 then holds. Note that, after a propagation distance $z$ a few times the decay length $l_{d} \equiv(1 / \sigma)$, one has $\left|S_{11}\right|^{2}=\left|S_{12}\right|^{2}=1 / 4$ and $\left|S_{13}\right|^{2} \simeq 1 / 2$, i.e. half of the injected light power has decayed into the slab, whereas the other half of light power is equally distributed into the two channel waveguides. The fact that the decay is not complete (fractional decay) indicates that a bound state embedded in the continuum does exist. When both waveguides $\mathrm{W}_{1}$ and $\mathrm{W}_{2}$ are initially excited with coherent fields of equal amplitudes but opposite sign, i.e. $c_{1}(0, \Omega)=-c_{2}(0, \Omega)=\sqrt{2 \pi} \delta(\Omega)$, one obtains $\psi(\rho, z)=\left[u_{1}(\rho)-u_{2}(\rho)\right] \exp \left(-i \Delta \beta_{0} z\right)$, i.e. the decay into the slab waveguide is fully suppressed. This is due to a destructive interference effect between different decay channels when $c_{2}=-c_{1}$ [see Eqs.(7) and (8)] and to the existence of a trapped state embedded in the continuum. Numerical examples of fractional light decay for single waveguide excitation, and of full decay suppression for simultaneous waveguide excitation in the trapped state, as obtained by a direct numerical analysis of Eq.(1) in the monochromatic regime, are shown in Fig.2. In the simulations, we assumed circular channel waveguides with a Gaussian index core profile of radius $r_{c}$ (at $1 / e$ ), and a step-index slab waveguide of thickness $a$. Equation (1) has been integrated by a standard split-step pseudospectral method with absorbing boundary conditions [9].

Generalization of light propagation in the nonmonochromatic case simply follows from the superposition principle. For instance, if waveguides $\mathrm{W}_{1}$ and $\mathrm{W}_{2}$ are excited at the input plane by two pulses with envelopes $r_{1}(t)$ and $r_{2}(t)$, from Eqs.(4), (10) and (11) it follows that the field envelope $\psi(\rho, z, t)$ can be cast in the form 


$$
\begin{array}{r}
\psi(\rho, z, t)=\left[S_{11}(z) r_{1}\left(t-\frac{z}{v_{g}}\right)+S_{21}(z) r_{2}\left(t-\frac{z}{v_{g}}\right)\right] u_{1}(\rho)+ \\
+\left[S_{12}(z) r_{1}\left(t-\frac{z}{v_{g}}\right)+S_{22}(z) r_{2}\left(t-\frac{z}{v_{g}}\right)\right] u_{2}(\rho)+\psi_{S}
\end{array}
$$

where $\psi_{S}=\psi_{S}(\rho, z, t)$ is the fractional part of the field tunnelled into the slab waveguide. In particular, let us consider two coherent pulses with the same envelope but phase reversed and delayed by an interval $\delta$, i.e. $r_{1}(t)=r(t)$ and $r_{2}(t)=-r(t-\delta)$. In this case, interference effects, leading to light trapping in waveguides $\mathrm{W}_{1}$ and $\mathrm{W}_{2}$ and full suppression of leakage into the slab waveguide $\mathrm{S}\left(\psi_{S} \simeq 0\right)$, occurs for $\delta=0$ or, approximately, for a delay $\delta$ much smaller than the characteristic pulse duration $\tau_{c}$. For $\delta$ larger than $\tau_{c}$, the two wave packets are not overlapped and behave as independent beams, leading to fractional decay. The latter scenario is also observed if the two pulses are temporally overlapped $(\delta=0)$ but incoherent, i.e. their phase difference changes randomly in time.

\section{QUANTUM DESCRIPTION AND NONCLASSICAL EFFECTS}

\section{A. Quantization procedure}

To describe propagation of nonclassical light in the coupled waveguide system, the classical paraxial wave field $\psi$ in Eq.(1) or, similarly, the classical c-numbers $c_{1}$, $c_{2}$ and $c_{k}$ in Eqs.(5-7), have to be replaced by quantummechanical operators satisfying suitable commutation relations, and different quantization procedures may be adopted. A first approach, which is well suited when the classical problem is formulated in terms of coupled-mode equations (5-7), is the input-output operator formalism commonly used for linear quantum-optical networks, either in the Heisenberg or in the Schrödinger pictures (see, for instance, [17, 25]). A second approach, suited when the classical problem in Eq.(1) is formulated as a propagative (rather than as an initial-value) problem, is to adopt a quantization procedure for the classical field $\psi$ as an evolution in space (rather than in time). In the Schrödinger picture, this leads to an evolution in space of a many-photon probability amplitude. Such a phenomenological approach, which will be adopted in the following analysis, has received a growing use and appreciation in quantum theories of optical solitons [26-28]; its consistency with standard canonical quantization procedure has been discussed in Ref.[27]. Similar procedures have been also developed to study, in the Heisenberg picture, paraxial propagation of nonclassical light and applied to problems of quantum imaging (see, for instance, 29]). The quantization procedure consists in writing the classical paraxial wave equation (1) in Hamiltonian form assuming the paraxial spatial coordinate $z$ as an independent variable [26]. Introducing the new field $\Pi=i \hbar \psi^{\dagger}$ and the Hamiltonian $H=\int d x d y d t \mathcal{H}$ with density

$$
\mathcal{H}=-\frac{i}{2 \beta}\left(\Pi_{x} \psi_{x}+\Pi_{y} \psi_{y}\right)-\frac{1}{v_{g}} \Pi \psi_{t}-i V(x, y) \Pi \psi,
$$

it readily follows that the Hamilton equations $\psi_{z}=$ $(\delta H / \delta \Pi), \Pi_{z}=-(\delta H / \delta \psi)$ yield Eq.(1) and its complex conjugate, so that $\Pi$ is canonically conjugated to $\psi$. Quantization is then accomplished by replacing the classical fields $\psi$ and $\Pi$ with the operators $\hat{\psi}(x, y, t)$ and $\hat{\Pi}=i \hbar \hat{\psi}^{\dagger}(x, y, t)$ satisfying the commutation relations $\left[\hat{\psi}(\rho, t), \hat{\psi}^{\dagger}\left(\rho^{\prime}, t^{\prime}\right)\right]=\delta\left(\rho-\rho^{\prime}\right) \delta\left(t-t^{\prime}\right)$ and $\left[\hat{\psi}(\rho, t), \hat{\psi}\left(\rho^{\prime}, t^{\prime}\right)\right]=\left[\hat{\psi}^{\dagger}(\rho, t), \hat{\psi}^{\dagger}\left(\rho^{\prime}, t^{\prime}\right)\right]=0$, where we have set $\rho=(x, y)$. By introducing the spectral decomposition $\hat{\psi}(\rho, t)=(2 \pi)^{-1 / 2} \int d \Omega \hat{\phi}(\rho, \Omega) \exp (-i \Omega t)$, the second-quantized Hamiltonian operator reads

$$
\hat{H}=\hbar \int d \rho d \Omega\left\{\frac{1}{2 \beta}\left(\hat{\phi}_{x}^{\dagger} \hat{\phi}_{x}+\hat{\phi}_{y}^{\dagger} \hat{\phi}_{y}\right)+\left[V(\rho)-\frac{\Omega}{v_{g}}\right] \hat{\phi}^{\dagger} \hat{\phi}\right\} .
$$

Note that in the spectral domain the following commutation relations hold for the operators $\hat{\phi}(\rho, \Omega)$ and $\hat{\phi}^{\dagger}(\rho, \Omega)$

$$
\begin{array}{r}
{\left[\hat{\phi}(\rho, \Omega), \hat{\phi}^{\dagger}\left(\rho^{\prime}, \Omega^{\prime}\right)\right]=\delta\left(\rho-\rho^{\prime}\right) \delta\left(\Omega-\Omega^{\prime}\right),} \\
{\left[\hat{\phi}(\rho, \Omega), \hat{\phi}\left(\rho^{\prime}, \Omega^{\prime}\right)\right]=\left[\hat{\phi}^{\dagger}(\rho, \Omega), \hat{\phi}^{\dagger}\left(\rho^{\prime}, \Omega^{\prime}\right)\right]=0 .}
\end{array}
$$

Note also that the field energy $U$ [Eq.(2)] corresponds to the operator $\hat{U}=\hbar \omega \int d \rho d \Omega \hat{\phi}^{\dagger}(\rho, \Omega) \hat{\phi}(\rho, \Omega)$. In the Schrödinger picture, the quantum field is described by a vector state $|\mathcal{Q}(z)\rangle$ which evolves according to

$$
i \hbar \frac{d|\mathcal{Q}\rangle}{d z}=\hat{H}|\mathcal{Q}\rangle
$$

whereas the operators $\hat{\phi}(\rho, \Omega)$ do not evolve with $z[30]$. The state $|\mathcal{Q}\rangle$ can be expanded in Fock space as $|\mathcal{Q}\rangle=$ $\sum_{n} a_{n}\left|f^{(n)}(\mathbf{q}, \boldsymbol{\Omega}, z)\right\rangle$, where the $n$-photon number state $\left|f^{(n)}\right\rangle$ is defined by (see, for instance, [26])

$$
\left|f^{(n)}\right\rangle=\int d \mathbf{q} d \boldsymbol{\Omega} \frac{f^{(n)}(\mathbf{q}, \boldsymbol{\Omega}, z)}{\sqrt{n !}} \hat{\phi}^{\dagger}\left(\rho_{1}, \Omega_{1}\right) \ldots . \hat{\phi}^{\dagger}\left(\rho_{n}, \Omega_{n}\right)|0\rangle
$$

and where we have set $\mathbf{q}=\left(\rho_{1}, \rho_{2}, \ldots\right), \boldsymbol{\Omega}=$ $\left(\Omega_{1}, \Omega_{2}, \ldots\right)$. The normalization conditions $\sum_{n}\left|a_{n}\right|^{2}=1$ and $\int d \mathbf{q} d \boldsymbol{\Omega}\left|f^{(n)}(\mathbf{q}, \boldsymbol{\Omega}, z)\right|^{2}=1$ are also assumed, which 
ensure that $\langle\mathcal{Q} \mid \mathcal{Q}\rangle=1$. Note that $\hat{U}\left|f^{(n)}\right\rangle=n \hbar \omega\left|f^{(n)}\right\rangle$, i.e. the Fock state $\left|f^{(n)}\right\rangle$ is obtained from the vacuum state $|0\rangle$ by creating $n$ photons with space-frequency weighting function $f^{(n)}$. The evolution equation for the weighting function $f^{(n)}$ is obtained by substituting Eqs.(18) and (21) into the Scrödinger equation (20) and using the commutation relations of field operators [Eqs.(19)]. One then obtains

$$
i \frac{\partial f^{(n)}}{\partial z}=\sum_{l=1}^{n}\left[-\frac{1}{2 \beta}\left(\frac{\partial^{2}}{\partial x_{l}^{2}}+\frac{\partial^{2}}{\partial y_{l}^{2}}\right)+V\left(\rho_{l}\right)-\frac{\Omega_{l}}{v_{g}}\right] f^{(n)} .
$$

Owing to the bosonic nature of photons, solely symmetric functions $f^{(n)}$ should be considered.

\section{B. Nonclassical effects with monochromatic beams}

Let us consider first the propagation of monochromatic fields, so that in Eqs.(18), (19), (21) and (22) we may disregard integration over different spectral components $\Omega$ and use a single renormalized bosonic creation operator $\hat{\phi}^{\dagger}(\rho)$ at frequency $\Omega=0$ satisfying the commutation relations [31] $\left[\hat{\phi}(\rho), \hat{\phi}^{\dagger}\left(\rho^{\prime}\right)\right]=\delta\left(\rho-\rho^{\prime}\right)$ and $\left[\hat{\phi}(\rho), \hat{\phi}\left(\rho^{\prime}\right)\right]=\left[\hat{\phi}^{\dagger}(\rho), \hat{\phi}^{\dagger}\left(\rho^{\prime}\right)\right]=0$, which replace Eq.(19). The simplest $n$-photon number state, denoted by $|g\rangle_{n}$, is obtained by assuming in Eq.(22) $f^{(n)}(\mathbf{q}, z)=$ $g\left(\rho_{1}, z\right) g\left(\rho_{2}, z\right) \ldots g\left(\rho_{n}, z\right)$, where the function $g(\rho, z)$ satisfies the classical wave optics equation

$$
i g_{z}=-\frac{1}{2 \beta}\left(g_{x x}+g_{y y}\right)+V(x, y) g
$$

with the normalization $\int d x d y|g(x, y, z)|^{2}=1$. In this case one has

$$
|g\rangle_{n}=\frac{1}{\sqrt{n !}}\left(\int d x d y g(x, y, z) \hat{\phi}^{\dagger}(x, y)\right)^{n}|0\rangle .
$$

Physically, this state describes the excitation of the optical system with an $n$-photon number state input beam with a spatial profile $g(x, y, 0)$ at the entrance plane $z=0$. The classic wave optics description of light propagation, discussed in Sec.II, is attained by considering a superposition of photon number states $\left|g_{n}\right\rangle$ with a Poissonian distribution with c-number $\alpha$, i.e. the coherent state $|g ; \alpha\rangle_{\text {coh }}$ defined by

$|g ; \alpha\rangle_{\mathrm{coh}}=\sum_{n=0}^{\infty} \frac{\exp \left(-|\alpha|^{2} / 2\right) \alpha^{n}}{n !}\left(\int d \rho g(\rho, z) \hat{\phi}^{\dagger}(\rho)\right)^{n}|0\rangle$.

One can readily show that the coherent state $|g ; \alpha\rangle_{\text {coh }}$ is an eigenstate of the field annihilation operator $\hat{\phi}(x, y)$ with eigenvalue $\alpha g(x, y, z)$, i.e. $\hat{\phi}(x, y)|g ; \alpha\rangle_{\text {coh }}=$ $\alpha g(x, y, z)|g ; \alpha\rangle_{\text {coh }}$, where $g(x, y, z)$ evolves according to the classical wave equation (23). Therefore the expectation value $\operatorname{coh}\langle g ; \alpha|\hat{\phi}(x, y)| g ; \alpha\rangle_{\mathrm{coh}}=\alpha g(x, y, z)$ yields the classical solution of the wave equation (23) for an input beam profile $\alpha g(x, y, 0)$. More generally, for a nonclassical state $|\mathcal{Q}\rangle$ obtained by an arbitrary superposition of photon number states $|g\rangle_{n}$ with amplitudes $a_{n}$, one can readily show that the expectation value of $\hat{\phi}^{\dagger}(x, y) \hat{\phi}(x, y)$ yields the classic wave optics intensity distribution, namely $\left\langle\mathcal{Q}\left|\hat{\phi}^{\dagger}(x, y) \hat{\phi}(x, y)\right| \mathcal{Q}\right\rangle=\langle n\rangle|g(x, y, z)|^{2}$, where $\langle n\rangle=\sum_{n} n\left|a_{n}\right|^{2}$ is the mean photon number of the input beam. The quantum aspects of nonclassical light for single beam excitation may be revealed when the statistics of photons trapped in waveguides $\mathrm{W}_{1}$ and $\mathrm{W}_{2}$ are considered. For example, let us consider excitation of waveguide $\mathrm{W}_{1}$ in its fundamental mode at the input plane $z=0$, so that $g(x, y, 0)=u_{1}(x, y)$, and let us compare the statistics of photons that remain in waveguide $\mathrm{W}_{1}$ when the input beam is a photon number state (nonclassical light) or a coherent state (classical light). According to the analysis of Sec.II.B, the wave amplitude $g$ evolves according to [see Eq.(15)]

$$
g(\rho, z)=S_{11}(z) u_{1}(\rho)+S_{12} u_{2}(\rho)+S_{13}(z) \theta(\rho, z),
$$

where $\left|S_{11}\right|^{2}+\left|S_{12}\right|^{2}+\left|S_{13}\right|^{2}=1$ and $\theta(\rho, z)$ defines the normalized spatial profile of the field tunnelled into the slab waveguide. If we introduce the operators

$$
\begin{aligned}
\hat{a}_{1}^{\dagger} & \equiv \int d x d y u_{1}(x, y) \hat{\phi}^{\dagger}(x, y) \\
\hat{a}_{2}^{\dagger} & \equiv \int d x d y u_{2}(x, y) \hat{\phi}^{\dagger}(x, y) \\
\hat{a}_{3}^{\dagger} & \equiv \int d x d y \theta(x, y, z) \hat{\phi}^{\dagger}(x, y)
\end{aligned}
$$

the commutation relations $\left[\hat{a}_{i}, \hat{a}_{k}^{\dagger}\right]=\delta_{i, k}$ and $\left[\hat{a}_{i}^{\dagger}, \hat{a}_{k}^{\dagger}\right]=$ $\left[\hat{a}_{i}, \hat{a}_{k}\right]=0(i, k=1,2,3)$ hold. Assuming that the vector state $|\mathcal{Q}\rangle$ is given by a superposition of photon number states $|g\rangle_{n}$ with amplitudes $a_{n}$, one can write

$$
|\mathcal{Q}\rangle=\sum_{n=0}^{\infty} \frac{a_{n}}{\sqrt{n !}}\left(S_{11} a_{1}^{\dagger}+S_{12} a_{2}^{\dagger}+S_{13} a_{3}^{\dagger}\right)^{n}|0\rangle .
$$

The joint photon distribution $P\left(n_{1} ; n_{2}, n_{3} ; z\right)$ to find $n_{1}$ photons in waveguide $\mathrm{W}_{1}, n_{2}$ photons in waveguide $\mathrm{W}_{2}$ and $n_{3}$ photons in the slab waveguide $\mathrm{S}$ is given by

$$
P\left(n_{1}, n_{2}, n_{3} ; z\right)=\left|\left\langle n_{1}, n_{2}, n_{3} \mid \mathcal{Q}\right\rangle\right|^{2}
$$

where we have set

$$
\left|n_{1}, n_{2}, n_{3}\right\rangle \equiv \frac{1}{\sqrt{n_{1} ! n_{2} ! n_{3} !}} \hat{a}_{1}^{\dagger n_{1}} \hat{a}_{2}^{\dagger n_{2}} \hat{a}_{3}^{\dagger n_{3}}|0\rangle
$$

The explicit expression of $P\left(n_{1}, n_{2}, n_{3} ; z\right)$ can be obtained by a double binomial expansion of the operator $\left(S_{11} a_{1}^{\dagger}+S_{12} a_{2}^{\dagger}+S_{13} a_{3}^{\dagger}\right)^{n}$ entering in Eq.(30). The marginal photon distribution $P_{1}\left(n_{1}, z\right)$ to find $n_{1}$ photons in waveguide $\mathrm{W}_{1}$ is then obtained as $P_{1}\left(n_{1} ; z\right)=$ $\sum_{n_{2}, n_{3}=0}^{\infty} P\left(n_{1}, n_{2}, n_{3} ; z\right)$, and reads explicitly (see, for instance, [17])

$$
P_{1}\left(n_{1} ; z\right)=\sum_{n=n_{1}}^{\infty}\left|a_{n}\right|^{2}\left(\begin{array}{c}
n \\
n_{1}
\end{array}\right) \kappa^{n_{1}}(1-\kappa)^{n-n_{1}}
$$


where $\kappa \equiv\left|S_{11}(z)\right|^{2}$. Similar expressions are obtained for the marginal photon distributions $P_{2}\left(n_{2}, z\right)$ and $P_{3}\left(n_{3}, z\right)$ for waveguides $\mathrm{W}_{2}$ and $S$ after setting $\kappa=\left|S_{12}(z)\right|^{2}$ and $\kappa=\left|S_{13}(z)\right|^{2}$, respectively. Let us now suppose that the input beam is a coherent state with mean photon number $\langle n\rangle=n_{0}$ and Poissonian distribution

$$
\left|a_{n}\right|^{2}=\frac{n_{0}^{n} \exp \left(-n_{0}\right)}{n !} .
$$

From Eq.(33) it follows that the marginal photon distribution $P_{1}\left(n_{1} ; z\right)$ remains Poissonian with mean photon number $\left\langle n_{1}\right\rangle=n_{0}\left|S_{11}(z)\right|^{2}$ that decays along the propagation distance $z$ according to the classical decay law given by Eq.(12). Conversely, if the input beam is a photon number state, i.e. $a_{n}=\delta_{n, n_{0}}$, from Eq.(33) it follows that the marginal photon distribution $P_{1}\left(n_{1} ; z\right)$ is given by the binomial distribution

$$
P_{1}\left(n_{1} ; z\right)=\left(\begin{array}{c}
n_{0} \\
n_{1}
\end{array}\right) \kappa^{n_{1}}(1-\kappa)^{n_{0}-n_{1}} \quad\left(n_{1} \leq n_{0}\right)
$$

$\left[P_{1}\left(n_{1} ; z\right)=0\right.$ for $\left.n_{1}>n_{0}\right]$ with photon mean $\left\langle n_{1}\right\rangle=n_{0}\left|S_{11}(z)\right|^{2}$ and variance $\left\langle\Delta n_{1}^{2}\right\rangle=\left|S_{11}(z)\right|^{2}[1-$
$\left.\left|S_{11}(z)\right|^{2}\right] n_{0}$. The binomial photon distribution highlights the particle-like behavior of photons undergoing the decay from waveguide $\mathrm{W}_{1}$ and it is analogous to that created by a beam splitter when excited by a photon number state in one port, and the vacuum state in the other one (see, for instance, [19]). The photons in the initially excited waveguide behave like independent classical particles and tunnel into the other waveguides is ruled by a Bernoulli trial (a coin toss) with a cumulative tunneling probability given by $1-\left|S_{11}(z)\right|^{2}$.

When the two waveguides $\mathrm{W}_{1}$ and $\mathrm{W}_{2}$ are simultaneously excited by two nonclassical independent beams, quantum signatures of light propagation resulting from quantum interference can be detected by photon coincidence measurements. To describe propagation of independent beams, let us notice that for a given set of normalized and orthogonal solutions $g_{1}(\rho, z), g_{2}(\rho, z), \ldots$ to the classic wave equation (23), one can construct the $n$-photon number state

$$
\left|g_{1}, g_{2}, \ldots\right\rangle_{n_{1}, n_{2}, \ldots}=\frac{1}{\sqrt{n_{1} ! n_{2} ! \ldots}}\left(\int d \rho g_{1}(\rho, z) \hat{\phi}^{\dagger}(\rho)\right)^{n_{1}} \times\left(\int d \rho g_{2}(\rho, z) \hat{\phi}^{\dagger}(\rho)\right)^{n_{2}} \times \ldots|0\rangle
$$

which describes excitation of the optical system with a set of independent beams with spatial profiles $g_{1}, g_{2}, \ldots$ carrying $n_{1}, n_{2}, \ldots$ photons $\left(n=n_{1}+n_{2}+\ldots\right)$. In particular, let us assume that at the input plane the waveguides $\mathrm{W}_{1}$ and $\mathrm{W}_{2}$ are excited in their fundamental modes by single photon number states, i.e. $g_{1}(\rho, 0)=u_{1}(\rho)$, $g_{2}(\rho, 0)=u_{2}(\rho)$ and

$$
|Q(z=0)\rangle=\left|u_{1}, u_{2}\right\rangle_{1,1}=\hat{a}_{1}^{\dagger} \hat{a}_{2}^{\dagger}|0\rangle,
$$

where the operators $\hat{a}_{1}^{\dagger}$ and $\hat{a}_{2}^{\dagger}$ are defined by Eqs.(27) and (28). According to the classical analysis of Sec.II.B, the waves $g_{1}$ and $g_{2}$ evolve according to

$$
\begin{aligned}
& g_{1}(\rho, z)=S_{11}(z) u_{1}(\rho)+S_{12}(z) u_{2}(\rho)+S_{13}(z) \theta(\rho, z) \\
& g_{2}(\rho, z)=S_{21}(z) u_{1}(\rho)+S_{22}(z) u_{2}(\rho)+S_{23}(z) \theta(\rho, z)
\end{aligned}
$$

with $S_{23}=S_{13}$ for symmetry reasons [32], $S_{12}=S_{21}$, $S_{11}=S_{22}$ [see Eqs.(12-14)], and $\left|S_{11}\right|^{2}+\left|S_{12}\right|^{2}+\left|S_{13}\right|^{2}=$ 1 for power conservation. From Eqs.(36), (38) and (39) it follows that the state vector of the system at the generic propagation distance $z$ is given by

$$
|\mathcal{Q}\rangle=\left(S_{11} \hat{a}_{1}^{\dagger}+S_{12} \hat{a}_{2}^{\dagger}+S_{13} \hat{a}_{3}^{\dagger}\right)\left(S_{21} \hat{a}_{1}^{\dagger}+S_{22} \hat{a}_{2}^{\dagger}+S_{23} \hat{a}_{3}^{\dagger}\right)|0\rangle
$$

where $\hat{a}_{3}^{\dagger}$ is defined in Eq.(29). It can be readily shown that the expectation value of the field intensity for the two-photon state (40) is given by the incoherent superposition

$$
\left\langle\mathcal{Q}\left|\hat{\phi}^{\dagger}(\rho) \hat{\phi}(\rho)\right| \mathcal{Q}\right\rangle=\left|g_{1}(\rho, z)\right|^{2}+\left|g_{2}(\rho, z)\right|^{2},
$$

the absence of interference being due to the lack of a definite phase relationship between the two photons (see, for instance, [33]). The two independent beams which excite the two waveguides $\mathrm{W}_{1}$ and $\mathrm{W}_{2}$ behave, therefore, as two incoherent classical fields, and therefore according to the analysis of Sec.II.B half of the light power tunnels, on average, into the slab waveguide $\mathrm{S}$ for propagation distances $z \gg l_{d}$. As the operators $\hat{a}_{1}^{\dagger}, \hat{a}_{2}^{\dagger}$ and $\hat{a}_{3}^{\dagger}$ create photons in waveguides $\mathrm{W}_{1}, \mathrm{~W}_{2}$ and $\mathrm{W}_{3}$, respectively [see Eqs.(27-29)], from Eq.(40) the joint photon distribution $P\left(n_{1}, n_{2}, n_{3} ; z\right)$ can be readily calculated, and its nonvanishing terms are explicitly given by 


$$
\begin{aligned}
& P(2,0,0 ; z)=P(0,2,0 ; z)=2\left|S_{11}\right|^{2}\left|S_{21}\right|^{2}=\frac{1}{8}[1-\exp (-4 \sigma z)]^{2} \\
& P(1,1,0 ; z)=\left|S_{11} S_{22}+S_{12} S_{21}\right|^{2}=\frac{1}{4}[1+\exp (-4 \sigma z)]^{2} \\
& P(1,0,1 ; z)=P(0,1,1 ; z)=\left|S_{11} S_{23}+S_{13} S_{21}\right|^{2}=\frac{1}{2} \exp (-4 \sigma z)[1-\exp (-4 \sigma z)] \\
& P(0,0,2 ; z)=2\left|S_{13}\right|^{2}\left|S_{23}\right|^{2}=\frac{1}{2}[1-\exp (-4 \sigma z)]^{2} .
\end{aligned}
$$

An important result is that, for propagation distances $z$ larger than the characteristic decay length $l_{d}=(1 / \sigma)$, the joint probability $P(1,0,1)$ to find one photon in waveguide $W_{1}$ and one in the slab waveguide $S$ vanishes, and similarly for $P(0,1,1)$ [see Eq.(44)]. Such a result indicates that, if one of the two initially injected photons tunnels into the slab waveguide, the other photon does the same, i.e. photon pairs bunch when decaying into the continuum. This is obviously a nonclassical effect similar to the two-photon Hong-Ou-Mandel quantum interference in a $50 \%$ beam splitter [18]. In our case, the vanishing of $P(1,0,1)$ [and similarly of $P(0,1,1)$ ] is related to a destructive interference between the probability amplitudes $S_{11} S_{23}$ and $S_{13} S_{21}$ entering in Eq.(44) which describe two possible paths for the photon pair.

\section{Nonclassical effects with wave packets}

In the previous subsection, we have considered propagation of nonclassical light in the monochromatic limit, however in practice nonclassical light such as that generated by spontaneous parametric down conversion in a nonlinear crystal is always polychromatic to some extent. Moreover, in experimental settings quantum interference such as the two-photon Hong-Ou-Mandel interference is typically revealed as a dip in intensity correlation (photon coincidence) measurements when the time delay between the two incoming wave packets is varied [18, 21]. It is therefore useful to extend the analysis of Sec.III.B to the case of polychromatic wave packets.

For the sake of definiteness, let us assume that waveguides $\mathrm{W}_{1}$ and $\mathrm{W}_{2}$ are excited in their fundamental spatial modes by two spectrally-narrow wave packets generated by spontaneous parametric down conversion in type-I nonlinear crystal. In this case, the vector state $|\mathcal{Q}\rangle$ at the input plane of the waveguiding system is a generalization of Eq.(37) and given by a superposition of two-photon states with a spectral function $C\left(\Omega_{1}, \Omega_{2}\right)$ (see, for instance, [18, 34])

$$
|\mathcal{Q}(z=0)\rangle=\int d \Omega_{1} d \Omega_{2} C\left(\Omega_{1}, \Omega_{2}\right) \hat{a}_{1}^{\dagger}\left(\Omega_{1}\right) \hat{a}_{2}^{\dagger}\left(\Omega_{2}\right)|0\rangle
$$

where the operators $\hat{a}_{1}^{\dagger}\left(\Omega_{1}\right)$ and $\hat{a}_{2}^{\dagger}\left(\Omega_{1}\right)$ are defined by

$$
\hat{a}_{1,2}^{\dagger}(\Omega)=\int d \rho u_{1,2}(\rho) \hat{\phi}^{\dagger}(\rho, \Omega) .
$$

The evolution of vector state along the propagation distance $z$ can be obtained by a straightforward extension of the analysis of Sec.III.B and reads explicitly

$$
\begin{aligned}
|\mathcal{Q}\rangle & =\int d \Omega_{1} d \Omega_{2} C\left(\Omega_{1}, \Omega_{2}\right) \exp \left[i\left(\Omega_{1}+\Omega_{2}\right) z / v_{g}\right]\left[S_{11}(z) \hat{a}_{1}^{\dagger}\left(\Omega_{1}\right)+S_{12}(z) \hat{a}_{2}^{\dagger}\left(\Omega_{1}\right)+S_{13}(z) \hat{a}_{3}^{\dagger}\left(\Omega_{1}\right)\right] \times \\
& \times\left[S_{21}(z) \hat{a}_{1}^{\dagger}\left(\Omega_{2}\right)+S_{22}(z) \hat{a}_{2}^{\dagger}\left(\Omega_{2}\right)+S_{23}(z) \hat{a}_{3}^{\dagger}\left(\Omega_{2}\right)\right]|0\rangle
\end{aligned}
$$

where

$$
\hat{a}_{3}^{\dagger}(\Omega)=\int d \rho \theta(\rho, z) \hat{\phi}^{\dagger}(\rho, \Omega)
$$

To study quantum interference effects with wave packets, and in particular the vanishing of the joint photon probability $P(1,0,1 ; z)=P(0,1,1 ; z)$ for $z \gg l_{d}$ found for monochromatic beams [Eq.(44)], let us introduce the integrated intensity correlation function at times $t_{1}$ and $t_{2}$ in waveguides $\mathrm{W}_{1}$ and $\mathrm{S}$ defined as 


$$
\mathcal{P}\left(t_{1}, t_{2} ; z\right)=\int_{\mathcal{A}_{1}} d \rho_{1} \int_{\mathcal{A}_{2}} d \rho_{2}\left\langle\mathcal{Q}\left|\hat{\psi}^{\dagger}\left(\rho_{2}, t_{2}\right) \hat{\psi}^{\dagger}\left(\rho_{1}, t_{1}\right) \hat{\psi}\left(\rho_{1}, t_{1}\right) \hat{\psi}\left(\rho_{2}, t_{2}\right)\right| \mathcal{Q}\right\rangle
$$

where $\mathcal{A}_{1}$ and $\mathcal{A}_{2}$ are two areas in the transverse $(x, y)$ plane surrounding waveguides $\mathrm{W}_{1}$ and $\mathrm{S}$, respectively. The correlation function $\mathcal{P}\left(t_{1}, t_{2}\right)$ is proportional to the joint probability of detecting one photon in waveguide $\mathrm{W}_{1}$ at time $t_{1}$, and one photon in waveguide $\mathrm{S}$ at time $t_{2}$, after a propagation distance $z$ from the input plane. In fact, $\left\langle\mathcal{Q}\left|\hat{\psi}^{\dagger}\left(\rho_{2}, t_{2}\right) \hat{\psi}^{\dagger}\left(\rho_{1}, t_{1}\right) \hat{\psi}\left(\rho_{1}, t_{1}\right) \hat{\psi}\left(\rho_{2}, t_{2}\right)\right| \mathcal{Q}\right\rangle$ is proportional to the joint probability of detecting one photon at point $\rho_{1}$ and time $t_{1}$, and one photon at point $\rho_{2}$ and time $t_{2}$ at the same propagation distance $z$. The integral over the areas $\mathcal{A}_{1}$ and $\mathcal{A}_{2}$ thus gives the joint probability of detecting one photon in waveguide $\mathrm{W}_{1}$ at time $t_{1}$, and one photon in waveguide $\mathrm{S}$ at time $t_{2}$. For photons generated by spontaneous parametric down conversion in type-I nonlinear crystal using a monochromatic pump beam at frequency $\omega_{p}=2 \omega$, the two-photon spectrum $C\left(\Omega_{1}, \Omega_{2}\right)$ of a pair of signal and idler photons can be expressed as 18, 34]

$$
C\left(\Omega_{1}, \Omega_{2}\right)=\delta\left(\Omega_{1}+\Omega_{2}\right) G\left(\Omega_{1}, \Omega_{2}\right) \exp \left[i \frac{\delta\left(\Omega_{1}-\Omega_{2}\right)}{2}\right]
$$

where $G\left(\Omega_{1}, \Omega_{2}\right)$ is the phase matching function. The last exponential term on the right hand side in Eq.(51) has been introduced to account for a possible time delay $\delta$ between signal and idler wave packets introduced by different optical paths from the crystal to waveguides $\mathrm{W}_{1}$ and $\mathrm{W}_{2}$. The phase matching function $G\left(\Omega_{1}, \Omega_{2}\right)$ is assumed to be a real-valued and symmetric function [i.e. $G\left(\Omega_{1}, \Omega_{2}\right)=G\left(\Omega_{2}, \Omega_{1}\right)$ ], peaked at around $\Omega_{1}=$ $\Omega_{2}=0$, with e.g. a Gaussian profile [18]. Taking into account that $\hat{\psi}(\rho, t)=(2 \pi)^{-1 / 2} \int d \Omega \hat{\phi}(\rho, \Omega) \exp (-i \Omega t)$, substitution of Eqs.(48) and (51) into Eq.(50), using the commutaion relations $\left[\hat{\phi}(\rho, \Omega), \hat{a}_{1,2}^{\dagger}\left(\Omega^{\prime}\right)\right]=u_{1,2}(\rho) \delta(\Omega-$ $\left.\Omega^{\prime}\right),\left[\hat{\phi}(\rho, \Omega), \hat{a}_{3}^{\dagger}\left(\Omega^{\prime}\right)\right]=\theta(\rho, z) \delta\left(\Omega-\Omega^{\prime}\right)$ and the relations $\int_{\mathcal{A}_{1}} d \rho\left|u_{1}(\rho)\right|^{2} \simeq 1, \int_{\mathcal{A}_{2}} d \rho|\theta(\rho, z)|^{2} \simeq 1$, $\int_{\mathcal{A}_{1,2}} d \rho\left|u_{2,1}(\rho)\right|^{2}=\int_{\mathcal{A}_{1}} d \rho|\theta(\rho, z)|^{2}=\int_{\mathcal{A}_{2}} d \rho\left|u_{2}(\rho)\right|^{2} \simeq$ 0 , after some lengthy but straightforward calculations one obtains

$$
\mathcal{P}\left(t_{1}, t_{2} ; z\right)=\left|r(\tau+\delta) S_{11}(z) S_{23}(z)+r(\tau-\delta) S_{13}(z) S_{21}(z)\right|^{2}
$$

where $\tau=t_{2}-t_{1}$ and where we introduced the real-valued correlation function $r(\tau)$ defined by

$$
r(\tau)=\frac{1}{2 \pi} \int d \Omega G(\Omega,-\Omega) \exp (-i \Omega \tau)
$$

In practice, coincidence measurements correspond to an integration of $\mathcal{P}\left(t_{1}, t_{2} ; z\right)$ with respect to the time dif- ference $\tau=t_{2}-t_{1}$ over the resolving coincidence time, which is typically much longer than the correlation time $\tau_{c}$ of $g(\tau)$. Integrating Eq.(52) with respect to $\tau$ from $-\infty$ to $\infty$ and taking into account that $S_{21}=S_{12}$ and $\left|S_{23}\right|^{2}=\left|S_{13}\right|^{2}=1-\left|S_{11}\right|^{2}-\left|S_{12}\right|^{2}$, the following expression for the correlation function $\mathcal{P}$ versus time delay $\delta$ is finally obtained

$$
\begin{aligned}
\mathcal{P}(\delta ; z)=\alpha[1 & \left.-\left|S_{11}(z)\right|^{2}-\left|S_{12}(z)\right|^{2}\right]\left[\left|S_{11}(z)\right|^{2}+\left|S_{21}(z)\right|^{2}+\right. \\
& \left.+2 \operatorname{Re}\left[S_{11}(z) S_{21}^{*}(z)\right] \frac{\int_{-\infty}^{\infty} d \tau r(\tau-\delta) r(\tau+\delta)}{\int_{-\infty}^{\infty} d \tau r^{2}(\tau)}\right]
\end{aligned}
$$

where $\alpha=\int_{-\infty}^{\infty} d \tau r^{2}(\tau)$ and the expression of the coefficients $S_{11}(z)$ and $S_{12}(z)$ are given by Eqs.(12) and (13). The behavior of $\mathcal{P}(\delta ; z)$ versus $\delta$ shows a charac- teristic dip at $\delta=0$ of width $\sim \tau_{c}$, which is analogous to the Hong-Ou-Mandel dip observed in two-photon interference from a beam splitter [18]. Far from the dip, $\mathcal{P}(\delta)$ 
reaches a constant value $\alpha\left(1-\left|S_{11}\right|^{2}-\left|S_{12}\right|^{2}\right)\left(\left|S_{11}\right|^{2}+\right.$ $\left.\left|S_{21}\right|^{2}\right)$. For $z$ larger than the characteristic decay length $l_{d}=1 / \sigma$, the correlation $\mathcal{P}(\delta ; z)$ vanishes at the dip $\delta=0$, i.e. when signal and idler wave packets are temporally overlapped, according to the analysis of Sec.III.B.

\section{CONCLUSIONS}

In this work we have theoretically investigated propagation of classical and nonclassical light in a waveguidebased photonic structure that provides an optical analogue of population trapping in the continuum encountered in atomic physics. For classical light waves, coupled-mode equation analysis, previously studied in Ref. [9], shows that Fano interference between different light leakage channels is responsible for the appearance of a trapped state embedded in the continuum. To study propagation of nonclassical light, a second quantization model for the scalar wave equation, in the paraxial and quasi-monochromatic approximations, has been adopted. As for input beam excitation in a coherent state the classical picture of light propagation is retrieved, quantum interference effects with no classical counterpart have been highlighted for photon number state excitation of the waveguide structure. In particular, the tendency of photon pairs to bunch when decaying into the continuum has been predicted. Such an effect, which is similar to the two-photon Hong-Ou-Mandel interference in a beam splitter [18], may be observed as a dip in photon coincidence measurements. Our results indicate that photonic structures originally designed to mimic with optical waves the classical analogues of quantummechanical phenomena encountered in atomic, molecular or condensed-matter physics [5], may exhibit themselves a strictly quantum behavior when single photon level is reached.
[1] S.G. Krivoshlykov, Quantum-Theoretical Formalism for Inhomogeneous Graded-IndexWaveguides (Akademie Verlag, Berlin, 1994).

[2] M.A.M. Marte and S. Stenholm, Phys. Rev. A 56, 2940 (1997).

[3] D. Dragoman, Prog. Opt. 42, 424 (2002).

[4] D. Dragoman and M. Dragoman, Quantum-Classical Analogies (Springer, Berlin, 2004).

[5] S. Longhi, "Quantum-optical analogies using photonic structures", Laser \& Photon. Rev. (to be published, 2009).

[6] A.M. Kenis, I. Vorobeichik, M. Orenstein, and N. Moiseyev, IEEE J. Quant. Electron. 37, 1321 (2001); E. Paspalakis, Opt. Commun. 258, 31 (2006); S. Longhi, Phys. Rev. E 73, 026607 (2006); S. Longhi, Phys. Lett. A 359, 166 (2006).

[7] S. Longhi, G. Della Valle, M. Ornigotti, and P. Laporta, Phys. Rev. B 76, 201101(R) (2007); G. Della Valle, M. Ornigotti, T. Toney Fernandez, P. Laporta, S. Longhi, A. Coppa, and V. Foglietti, Appl. Phys. Lett. 92, 011106 (2008); Y. Lahini, F. Pozzi, M. Sorel, R. Morandotti, D.N. Christodoulides, and Y. Silberberg, Phys. Rev. Lett. 101, 193901 (2008).

[8] D.D. Smith, H. Chang, K.A. Fuller, A.T. Rosenberger, and R.W. Boyd, Phys. Rev. A 69, 063804 (2004); A. Naweed, G. Farca, S.I. Shopova, and A.T. Rosenberger, Phys. Rev. A 71, 043804 (2005); Q. Xu, S. Sandhu, M. L. Povinelli, J. Shakya, S. Fan, and M. Lipson, Phys. Rev. Lett. 96, 123901 (2006); P. Ginzburg and M. Orenstein, Opt. Express 14, 11312 (2006).

[9] S. Longhi, Phys. Rev. A 78, 013815 (2008).

[10] S. Longhi, "Optical analogue of coherent population trapping via a continuum in optical waveguide arrays", J. Mod. Opt. (to be published) DOI: 10.1080/09500340802187373.

[11] D.C. Marinica, A.G. Borisov, S.V. Shabanov, Phys. Rev. Lett. 100, 183902 (2008).

[12] P.E. Coleman and P.L. Knight, J. Phys. B 15, L235
(1982); P.E. Coleman, P.L. Knight, and K. Burnett, Opt. Commun. 42, 171 (1982); B.N. Dai and P. Lambropoulos, Phys. Rev. A 36, 5205 (1987); S. Cavalieri, F.S. Pavone, and M. Matera, Phys. Rev. Lett. 67, 3673 (1991); T. Nakajima and P. Lambropoulos, Opt. Commun. 118, 40 (1995); T. Halfmann, L.P. Yatsenko, M. Shapiro, B.W. Shore, and K. Bergmann, Phys. Rev. A 58, R46 (1998); E. Paspalakis, M. Protopapas, and P.L. Knight, J. Phys. B 31, 775 (1998).

[13] P.L. Knight, M.A. Lander, and B. J. Dalton, Phys. Rep. 190, 1 (1990).

[14] U. Fano, Phys. Rev. 124, 1866 (1961).

[15] S. Fan, P.R. Villeneuve, J.D. Joannopoulos, and H.A. Haus, Phys. Rev. Lett. 80, 960 (1998); S. Fan, P.R. Villeneuve, J.D. Joannopoulos, M.J. Khan, C. Manolatou, and H.A. Haus, Phys. Rev. B 59, 15882 (1999); Y. Xu, Y. Li, R.K. Lee, and A. Yariv, Phys. Rev. E 62, 7389 (2000); S. Fan and J. D. Joannopoulos, Phys. Rev. B 65, 235112 (2002); P. Chak, S. Pereira, and J.E. Sipe, Phys. Rev. B 73, 035105 (2006).

[16] A.E. Miroshnichenko, S.F. Mingaleev, S. Flach, and Yu.S. Kivshar, Phys. Rev. E 71, 036626 (2005); A.E. Miroshnichenko and Y.S. Kivshar, Phys. Rev. E 72, 056611 (2005); M.I. Tribelsky, S. Flach, A.E. Miroshnichenko, A.V. Gorbach, and Yu.S. Kivshar, Phys. Rev. Lett. 100, 043903 (2008).

[17] W.K. Lai, V. Bužek, and P.L. Knight, Phys. Rev. A 43, 6323 (1991).

[18] C.K. Hong, Z.Y. Ou, and L. Mandel, Phys. Rev. Lett. 59, 2044 (1987).

[19] R.A. Campos, B.E.A. Saleh, and M.C. Teich, Phys. Rev. A 40, 1371 (1989).

[20] J. Janszky, C. Sibilia, and M. Bertolotti, J. Mod. Opt. 35, 1757 (1988); A. Bandyopadhyay and J. Rai, Opt. Commun. 140, 41 (1997); M.S. Kim, W. Son, V. Bužek, and P.L. Knight, Phys. Rev. A 65, 032323 (2002); A. Rai, G.S. Agarwal, and J.H.H. Perk, Phys. Rev. A 78, 042304 (2008). 
[21] A. Politi, M.J. Cryan, J.G. Rarity, S. Yu, and J.L. O'Brien, Science 320, 646 (2008).

[22] Y. Bromberg, Y. Lahini, R. Morandotti, and Y. Silberberg, arXiv:0807.3938v1 [quant-ph] (2008).

[23] S. Longhi, Phys. Rev. Lett. 101, 193902 (2008).

[24] T.A.B. Kennedy and E.M. Wright, Phys. Rev. A 38, 212 (1988).

[25] U. Leonhardt, Rep. Prog. Phys. 66, 1207 (2003); J. Skaar, J.C.G. Escartin, and H. Landro, Am. J. Phys. 72, 1385 (2004).

[26] Y. Lai and H.A. Haus, Phys. Rev. A 40, 844 (1989); E.M. Wright, Phys. Rev. A 43, 3836 (1991); P.L. Hagelstein, Phys. Rev. A 54, 2426 (1996)

[27] A.B. Matsko and V.V. Kozlov, Phys. Rev. A 62, 033811 (2000).

[28] B. Yurke and M. J. Potasek, J. Opt. Soc. Am. B 6, 1227 (1989); C. Sibilia, V. Schiavone, M. Bertolotti, R. Horak, and J. Perina, J. Opt. Soc. Am. B 11, 2175 (1994); B Crosignani, P Di Porto and A Treppiedi, Quantum Semiclass. Opt. 7, 73 (1995); J.M. Fini, P.L. Hagelstein, and H. A. Haus, Phys. Rev. A 60, 2442 (1999); D. Rand, K. Steiglitz, and P.R. Prucnal, Phys. Rev. A 71, 053805 (2005).

[29] H.I. Yuen, and J.H. Shapiro, IEEE Trans. Inf. Theory IT-24, 657 (1978); M.I. Kolobov, Rev. Mod. Phys. 71, 1539 (1999); M. Bache, E. Brambilla, A. Gatti, and L.A. Lugiato, Phys. Rev. A 70, 023823 (2004); A. Aiello and J.P. Woerdman, Phys. Rev. A 72, 060101(R) (2005); M. Tsang and D. Psaltis, Phys. Rev. A 73, 013822 (2006).

[30] In the Heisenberg picture, the state vector of the system would be constant whereas the operator $\hat{\phi}$ evolve accord- ing to $i \hbar(d \hat{\phi} / d z)=[\hat{\phi}, \hat{H}]$. One can easily check that, with the Hamiltonian operator given by Eq.(18), the evolution equation of the operator $\hat{\phi}$ is precisely Eq.(3), where the c-field $\phi$ is replaced by the operator $\hat{\phi}$.

[31] More precisely, the monochromatic limit is attained by assuming in Eqs.(21) and $(22) f^{(n)}(\mathbf{q}, \boldsymbol{\Omega}, z)=$ $g^{(n)}(\mathbf{q}, z) h\left(\Omega_{1}\right) h\left(\Omega_{2}\right) \ldots h\left(\Omega_{n}\right)$, where $h(\Omega)$ is a spectrally narrow function at around $\Omega=0$ normalized such that $\int d \Omega|h(\Omega)|^{2}=1$, and $g^{(n)}(\mathbf{q}, z)$ satisfies Eq.(22) with $\Omega_{l}=0$. The operator $\hat{\phi}(\rho)$ is then formally defined as $\hat{\phi}(\rho)=\int d \Omega h(\Omega) \hat{\phi}(\rho, \Omega)$. The $n$-photon number state $\left|f^{(n)}\right\rangle$, given by Eq. (21), takes then the simplified form $\left|f^{(n)}\right\rangle=\left(1 /(\sqrt{n !}) \int d \mathbf{q} g^{(n)}(\mathbf{q}, z) \hat{\phi}^{\dagger}\left(\rho_{1}\right) \hat{\phi}^{\dagger}\left(\rho_{2}\right) \ldots \hat{\phi}^{\dagger}\left(\rho_{n}\right)|0\rangle\right.$.

[32] As the waveguides $\mathrm{W}_{1}$ and $\mathrm{W}_{2}$ are symmetrically placed at opposite sides from the slab waveguide $\mathrm{S}$, if $\left(c_{1}(z), c_{2}(z), c_{k}(z)\right)$ is a solution to Eqs.(5-7) with initial condition $c_{1}(0)=1, c_{2}(0)=0$ and $c_{k}(0)=0$, then $\left(c_{2}(z), c_{1}(z), c_{k}(z)\right)$ is the solution to Eqs.(5-7) with reversed initial condition $c_{1}(0)=0, c_{2}(0)=1$ and $c_{k}(0)=0$. Light tunnelled into the slab waveguide $\mathrm{S}$ is thus given by the same superposition of continuous modes $\left(\sim \int d k c_{k}(z) u_{k}(\rho)\right)$ for the two different initial conditions. This means that the last two terms on the right hand sides of Eqs.(38) and (39) are equal each other.

[33] R. Ghosh and L. Mandel, Phys. Rev. Lett. 59, 1903 (1987); L. Mandel, Rev. Mod. Phys. 71, S274 (1999).

[34] K. Wang, J. Phys. B 39, R293 (2006); W.P. Grice and I.A. Walmsley, Phys. Rev. A 56, 1627 (1997). 


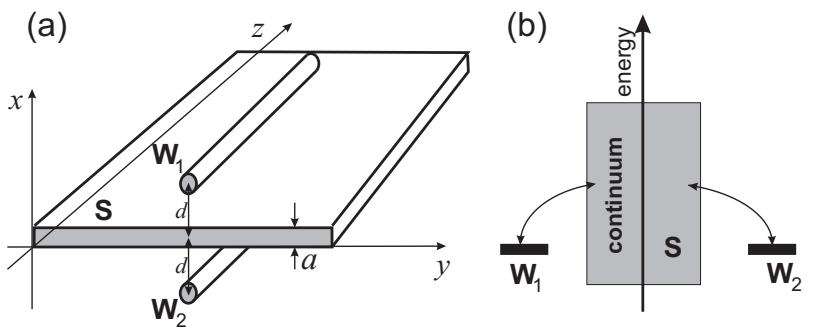

FIG. 1: (a) Schematic of the coupled waveguide system composed by two equal single-mode channel waveguides $\mathrm{W}_{1}$ and $\mathrm{W}_{2}$ side-coupled to a slab waveguide $\mathrm{S}$. (b) The quantum mechanical analogue, showing the decay of two bound states coupled to a common continuum. 
(a)
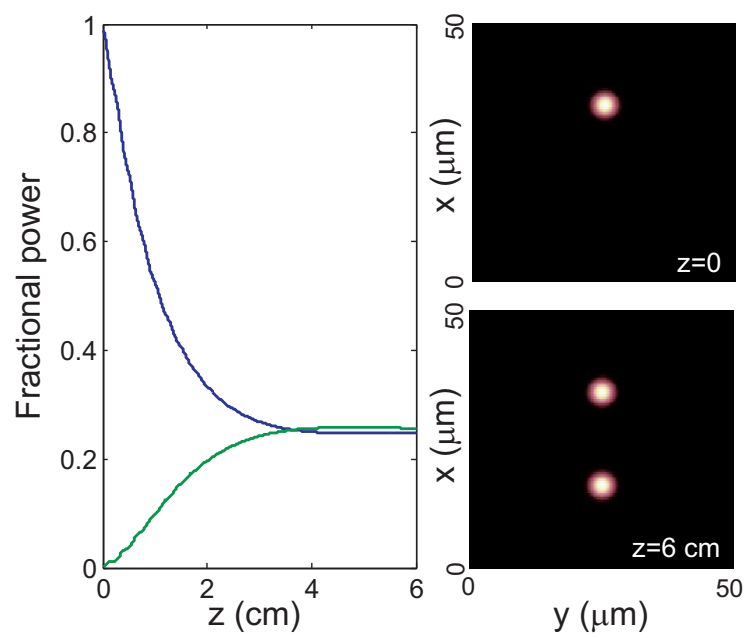

(b)

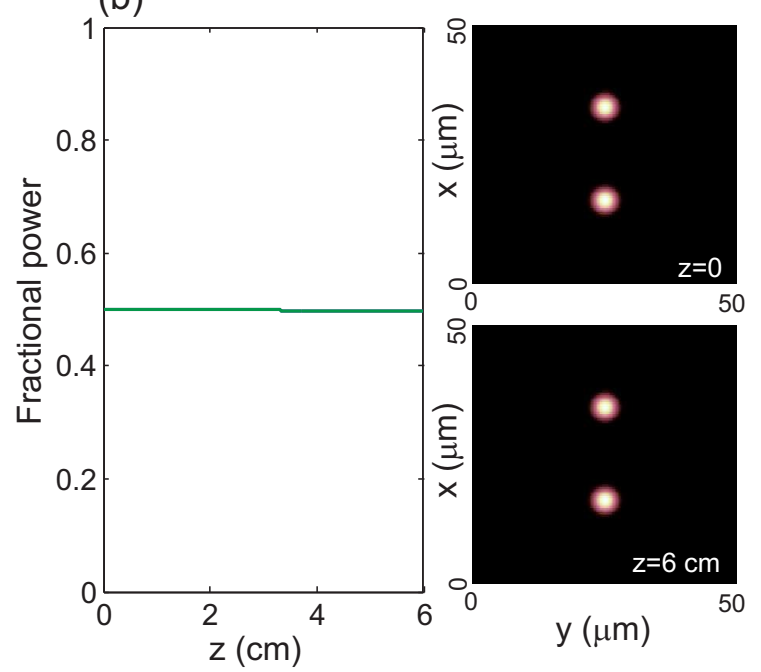

FIG. 2: (color online) Behavior of fractional light power trapped in channel waveguides $\mathrm{W}_{1}$ (blue curve) and $\mathrm{W}_{2}$ (green curve) versus propagation distance in a $L=6$-cm-long structure and corresponding transverse light intensity distributions at the input $(z=0)$ and output $(z=6 \mathrm{~cm})$ planes, as obtained by numerical simulations of the wave equation (1) for two different excitation conditions. In (a) waveguide $\mathrm{W}_{1}$ is excited in its fundamental mode, leading to fractional decay. In (b) the trapped state is excited, corresponding to full suppression of light leakage into the slab S. Parameter values used in the simulations are: $\lambda=980 \mathrm{~nm}, n_{s}=1.52$, $\Delta n_{S}=0.0049, \Delta n_{g}=0.01, d=9 \mu \mathrm{m}, r_{c}=2 \mu \mathrm{m}$, and $a=4 \mu \mathrm{m}$. 